\title{
Solutal Marangoni flows of miscible liquids drive transport without surface contamination
}

\author{
Hyoungsoo Kim ${ }^{1,2 \star}$, Koen Muller ${ }^{3}$, Orest Shardt ${ }^{1 \dagger}$, Shahriar Afkhami ${ }^{4}$ and Howard A. Stone ${ }^{1 \star}$
}

\begin{abstract}
Mixing and spreading of different liquids are omnipresent in nature, life and technology, such as oil pollution on the sea $^{1,2}$, estuaries ${ }^{3}$, food processing ${ }^{4}$, cosmetic and beverage industries $^{5,6}$, lab-on-a-chip devices ${ }^{7}$, and polymer processing ${ }^{8}$. However, the mixing and spreading mechanisms for miscible liquids remain poorly characterized. Here, we show that a fully soluble liquid drop deposited on a liquid surface remains as a static lens without immediately spreading and mixing, and simultaneously a Marangoni-driven convective flow is generated, which are counterintuitive results when two liquids have different surface tensions. To understand the dynamics, we develop a theoretical model to predict the finite spreading time and length scales, the Marangoni-driven convection flow speed, and the finite timescale to establish the quasi-steady state for the Marangoni flow. The fundamental understanding of this solutal Marangoni flow may enable driving bulk flows and constructing an effective drug delivery and surface cleaning approach without causing surface contamination by immiscible chemical species.
\end{abstract}

When a sessile oil drop is released on top of a water surface, it spreads until a monolayer is achieved ${ }^{9}$, because the liquids are immiscible, as shown in Fig. 1a. In contrast, if a water drop is placed on a water surface, it shows a cascade of coalescence events and the liquids are rapidly mixed (Fig. $1 b)^{10}$. In contrast with these two configurations, we captured unexpected mixing and spreading features between fully miscible liquids. When a drop of alcoholfor example, isopropanol (IPA) - is placed on a water surface, it spontaneously generates a Marangoni convective flow along the outward radial direction and we observed that there is a static liquid lens in the middle (Fig. 1c and Supplementary Fig. 1), even though these two liquids are infinitely miscible. Here, we discuss solutal Marangoni effects in fully miscible liquids to explain the finite size lens and the associated flow (more details are provided in Supplementary Videos 1-3).

To visualize the spreading and mixing pattern of a miscible liquid drop, IPA (volume $V=7.2 \pm 0.2 \mu \mathrm{l}$ ), placed on a water bath $(400 \mathrm{ml}$ deionized (DI) water in an 196-mm-diameter Petri dish with depth $H=14 \mathrm{~mm}$ ), we used time-resolved particle tracking velocimetry (PTV) and high-speed schlieren measurement techniques (Supplementary Information). For PTV experiments, we seeded polystyrene particles (diameter $=100 \mu \mathrm{m}$ ) in solution and recorded the particle motion from top and side views.

IPA is less dense than water and therefore the sessile drop floats on the surface. The drop initially spreads out and quickly achieves a static central lens with a near constant diameter $2 R$ (see Fig. $1 \mathrm{c}$ and
Supplementary Figs 5-7) during which the IPA continuously leaks at the boundary (Fig. 1c, Supplementary Fig. 1 and Supplementary Videos 3 and 4). The inset of Fig. 2a shows a top view of a schlieren pattern, that is, an interfacial turbulence structure representing the mass transfer between the phases ${ }^{11,12}$. Due to the Marangoni-driven mixing, the spreading coefficient $S$ (Fig. 2a) becomes zero ${ }^{13}$, that is,

$$
S \approx \gamma_{\text {local }}-\left(\gamma_{12}+\gamma_{23}\right) \simeq 0
$$

where the interfacial tension $\left(\gamma_{23}\right)$ between IPA and water is extremely small compared to other surface tensions ${ }^{13}$. Beneath this mixing zone, we observed from the side flow patterns in the experiment (Fig. 2b and Supplementary Video 6): circulating vortices are located near the edge of the lens.

To measure the spreading speed over the water surface, we seeded $100 \mu \mathrm{m}$ hydrophobic tracer particles in the IPA drop and tracked the particle motion from the top. Here, we assumed that the particles follow the spreading behaviour of the IPA liquid (Fig. 2c,d and details in Methods). The PTV method was used to obtain the flow speed (Supplementary Information and Supplementary Video 7). As indicated by the red arrows sketched in Fig. 2c, starting from the middle of the drop, we observe that the interfacial flow speed rapidly increases to a maximum at the edge of the static lens, and then decreases monotonically with distance beyond the drop. While the IPA propagates along the water surface, after finite timescales $(\tau)$ to establish a quasi-steady state, the maximum flow speed $(U)$ and finite lens size $(R)$ remain approximately constant (deviations are typically less than 10\%). The circulating flow in the bath brings fresh water from the bulk to the interface (see Fig. 2b), which maintains a constant surface tension gradient near the contact line (that is, air, IPA and water meet together), as sketched in Fig. 3a. Moreover, such flows would act to effectively sweep surface-bound contaminants away from the location of the drop (Supplementary Video 9).

In the literature, a qualitatively similar flow pattern of a radially outward velocity profile has been reported-for example, the spreading of injected soluble surfactant ${ }^{14}$ or continuous injection of partially (or fully) miscible liquid on water ${ }^{15-18}$-although the quantitative features (power laws) are distinct owing to the different physics of the spreading miscible fluids studied here. For example, for the soluble surfactant spreading case, the velocity profile shows a power-law behaviour ${ }^{14}, u \sim r^{-1 / 3}$ where $\Delta \gamma$ is constant at the leading edge. For the miscible liquids spreading case, an understanding of the spreading and mixing mechanism is still lacking, although particular mixing features were captured ${ }^{19,20}$. Therefore, to understand this case, we performed many different

${ }^{1}$ Department of Mechanical and Aerospace Engineering, Princeton University, Princeton, New Jersey 08544, USA. ${ }^{2}$ Department of Mechanical Engineering, KAIST, Daejeon 34141, South Korea. ${ }^{3}$ Process and Energy, Delft University of Technology, 2628 CA Delft, the Netherlands. ${ }^{4}$ Department of Mathematical Sciences, New Jersey Institute of Technology, Newark, New Jersey 07102, USA. Present address: Bernal Institute and School of Engineering, University of Limerick, Castletroy, Limerick V94 T9PX, Ireland *e-mail: hshk@kaist.ac.kr; hastone@princeton.edu 

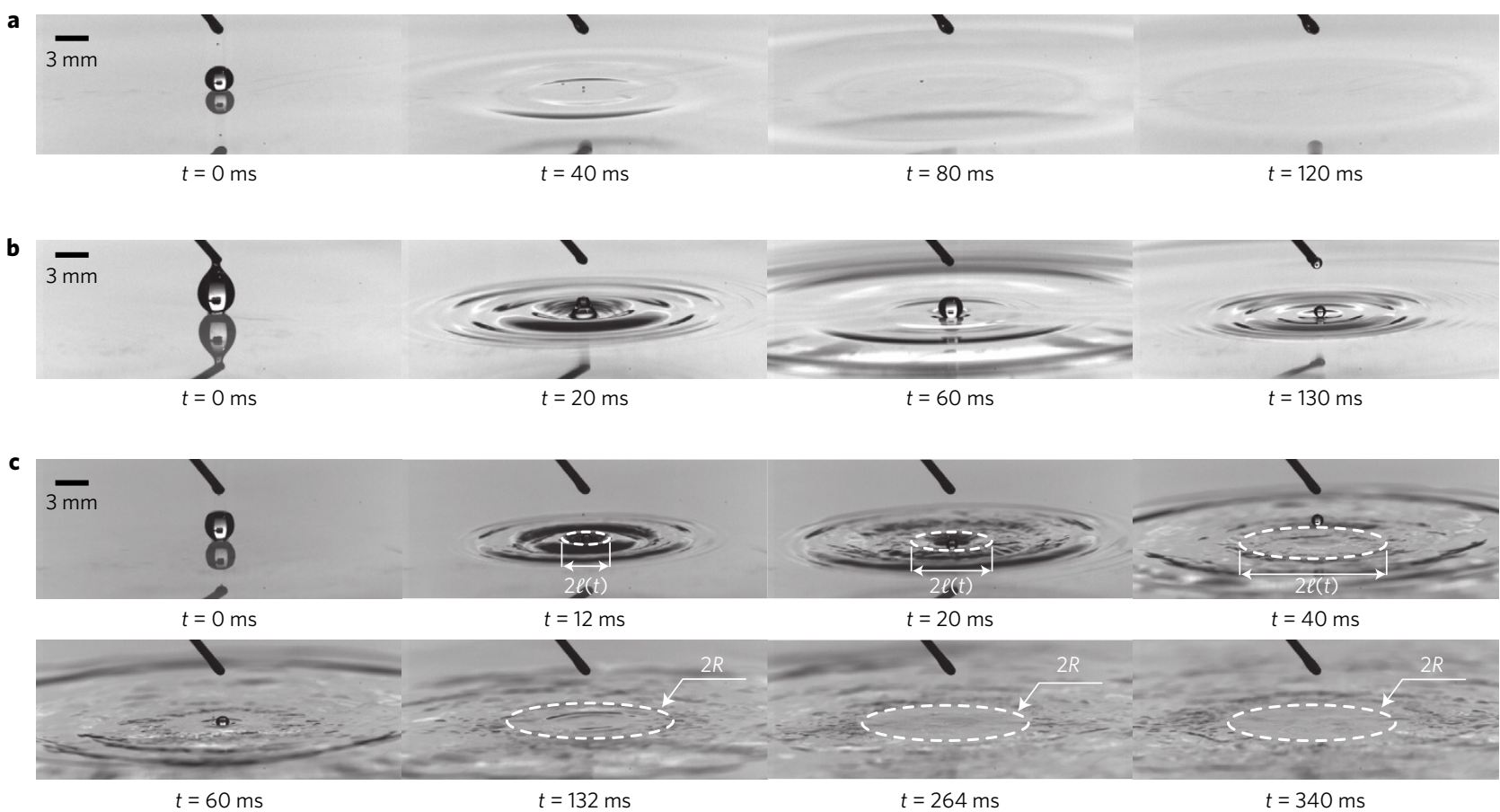

Figure 1 | Liquid drop mixing and spreading on a water surface. a, An immiscible liquid drop (silicone oil, 5 cSt) spreading on a water surface (initial drop radius, $a=1.2 \mathrm{~mm})$. b. A water drop on a water surface $(a=1.5 \mathrm{~mm}$ ). $\mathbf{c}$, A drop of isopropanol (fully miscible) mixing and spreading on a water surface $(a=1.2 \mathrm{~mm})$. The dashed circles indicate the rim of the deposited droplet and the radius is $\ell(t)$, which is confirmed with particle tracking velocimetry and background-oriented schlieren results (see Fig. 2c, Supplementary Fig. 6 and Supplementary Information). The static lens diameter (2R) is achieved at times approximately $\mathrm{O}(10 \mathrm{~ms})$. For more details, see Supplementary Videos 1-4.
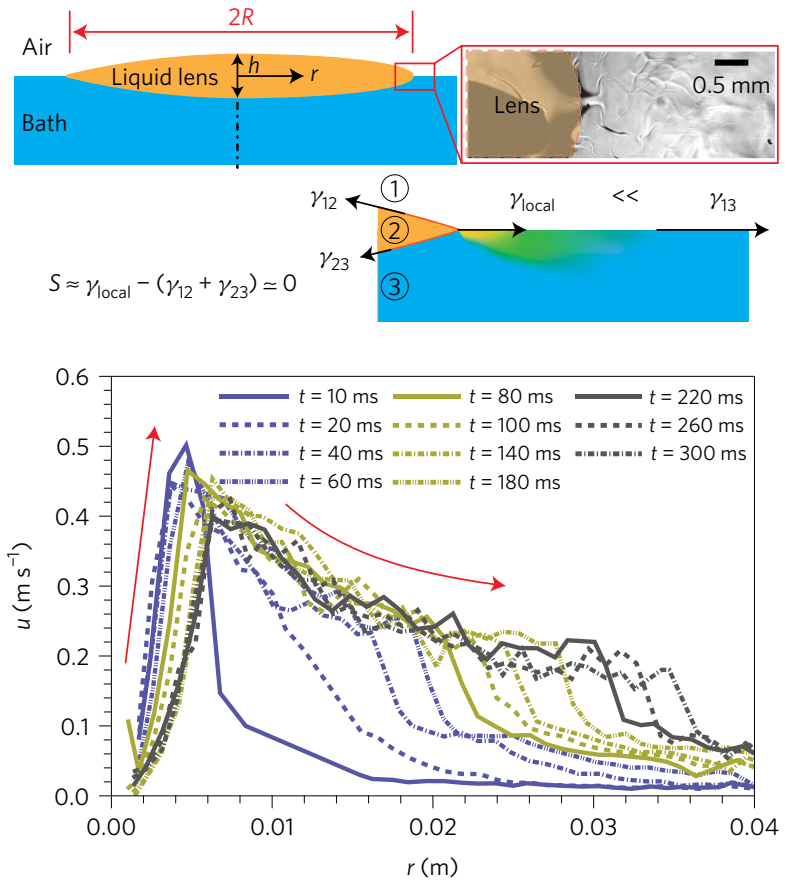

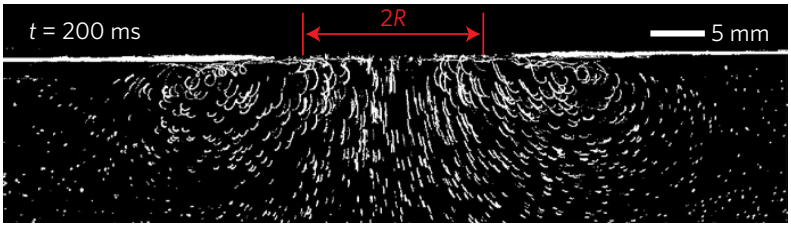

d

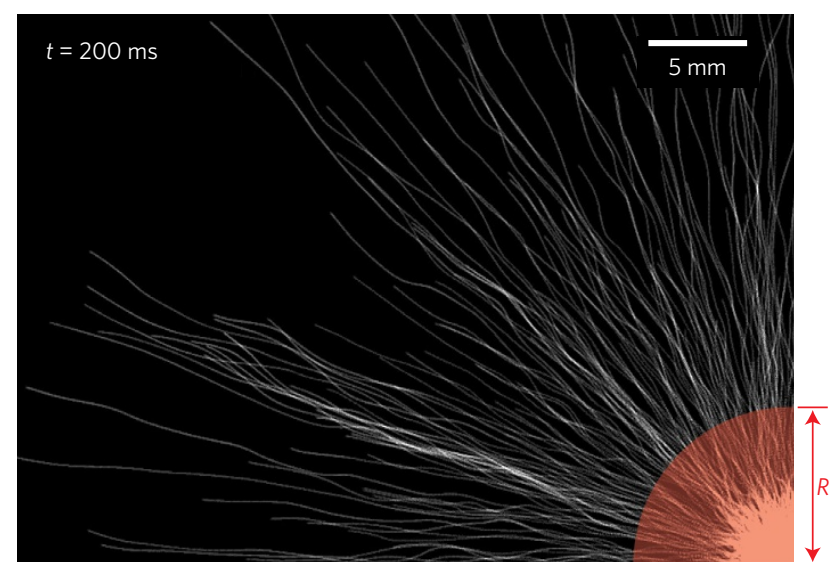

Figure 2 | Spreading and mixing between an IPA droplet and a water bath. a, Schematic of the interfacial tensions of a static liquid lens of IPA on water where the spreading coefficient $S$ becomes zero (phases: air, circled one; drop liquid, circled two; and water, circled three). The liquid lens diameter is $2 R$. Inset, schlieren high-speed image from a top view. For more details, see Supplementary Videos 4 and 5 . b, Side-view trajectories of particles

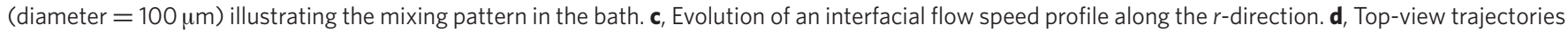
of particles representing the spreading pattern where $100 \mu \mathrm{m}$ particles are seeded in the droplet. For more details, see Supplementary Videos $6-8$.

experiments and report results for 12 different combinations of miscible liquids (Table 1) and by adding different surfactantssodium dodecyl sulfate (SDS) and cetyl trimethyl ammonium bromide $(\mathrm{CTAB})$ - into water. We measured the interfacial flow speed profile during the quasi-steady regime. As shown in Fig. 3c and $\mathrm{d}$, respectively, the maximum Marangoni convective flow speed 
a
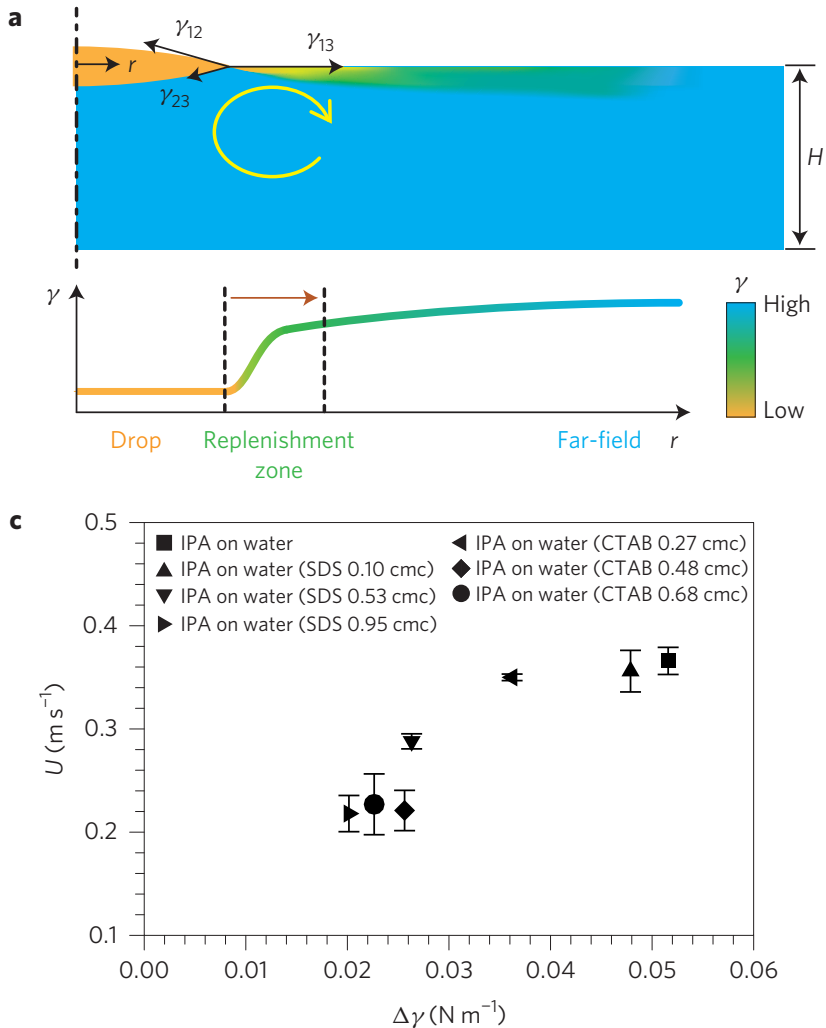

e

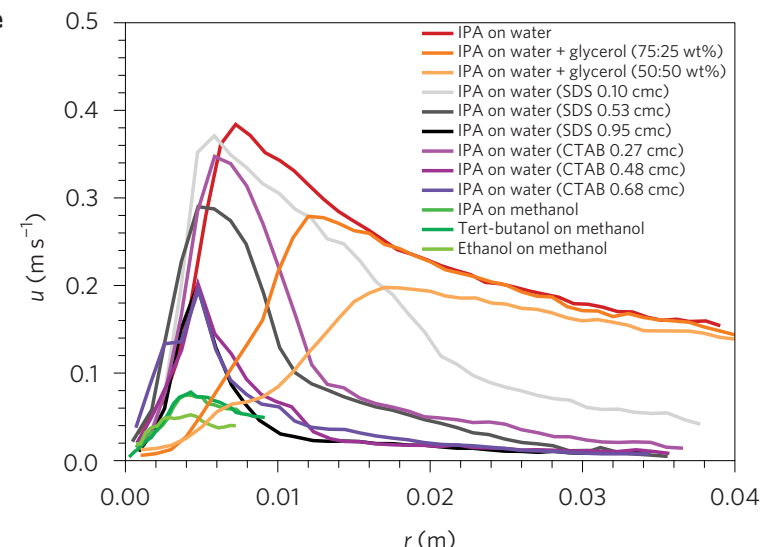

b

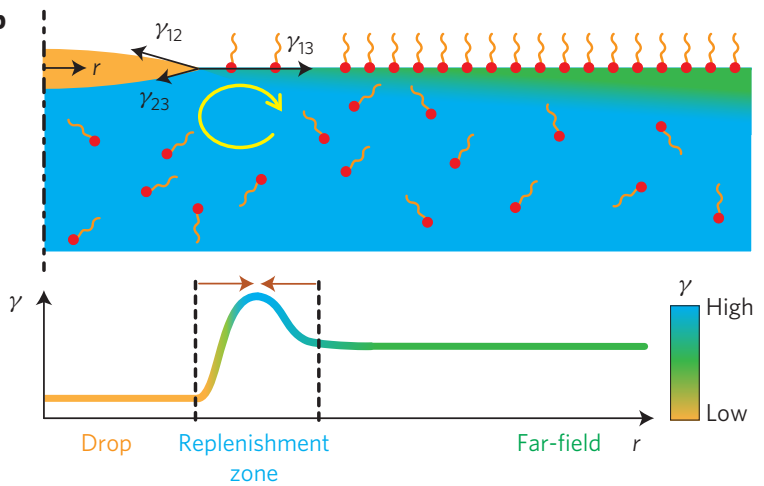

d

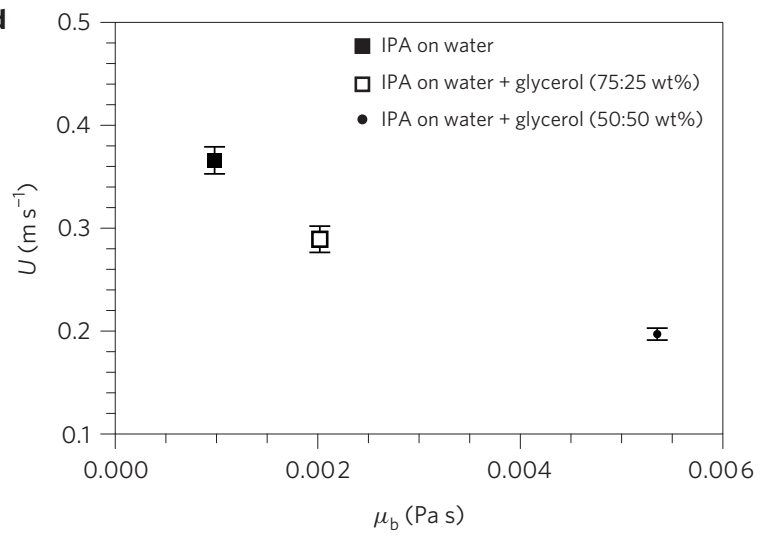

f

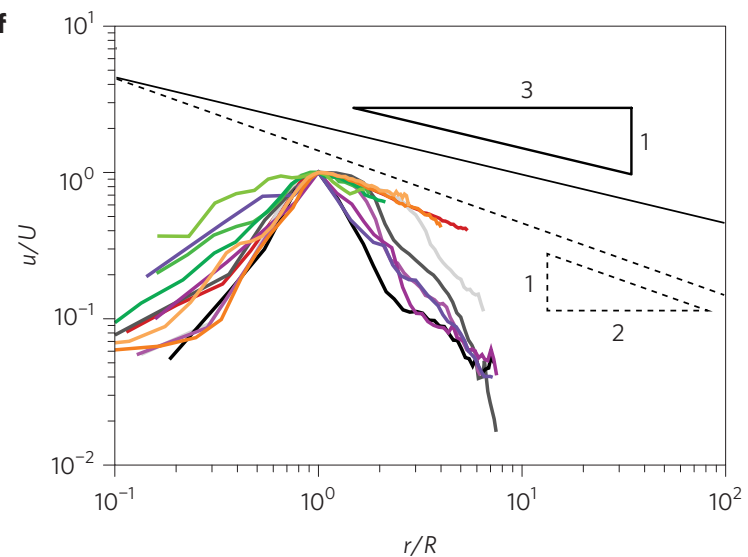

Figure 3 | A fully miscible liquid droplet spreading along the interface of a liquid bath in a quasi-steady state. a, Schematic of an interfacial tension ( $\gamma$ ) profile of a deposited fully miscible drop on a pure solution of higher $\gamma$. $\mathbf{b}$, Schematic of an interfacial surfactant distribution and an interfacial tension profile on a surfactant-contaminated solution. $\mathbf{c}$, The effect of the surface tension difference on the maximum Marangoni flow speed (U). d, The effect of the dynamic viscosity on the maximum Marangoni flow speed $(U)$ at the rim $(r \approx R)$ of the static lens drop. For $\mathbf{c}$ and $\mathbf{d}$, the error bars are obtained from the standard deviation of the maximum velocity $(U)$ magnitudes during the quasi-steady regime. e, Interfacial flow speed $(u)$ profiles in the $r$-direction for 12 different liquid systems. f, Rescaled interfacial flow speed profiles $u / U$ as a function of $r / R$, where $U$ is the maximum speed and $R$ is the radius of the static lens.

$(U)$ is proportional to the surface tension difference between the two liquids $\left(\Delta \gamma=\gamma_{13}-\gamma_{12}\right)$ and inversely proportional to the dynamic viscosity of the liquid bath. To investigate the velocity profile (Fig. 3e), we plotted $u / U$ versus $r / R$ for all of the tested cases (Fig. 3f). The fully miscible case without surfactants shows $u / U \sim(r / R)^{-1 / 2}$ (see the dashed line in Fig. 3f). $\Delta \gamma$ at the leading front beyond the static lens decreases along the $r$-direction as a result of mixing between the ejected alcoholic liquid (IPA, ethanol and tert-butanol) and the liquid bath (water, water-glycerol mixtures and methanol) while spreading.

For the surfactant-contaminated liquid bath, the surface tension profile along the interface is expected to change as sketched in Fig. 3b, as a consequence of a Marangoni-driven circulating flow and non-uniform surfactant distribution. Below the critical micelle concentration $(\mathrm{cmc})$, the surfactant concentrations in the adsorbed layer (near the air-water interface) and in the bulk are different ${ }^{21}$. The circulating flow delivers bulk liquid that has a low surfactant concentration (relatively high surface tension) compared to that in the adsorbed layer, and hence two opposite Marangoni effects occur in a replenishment zone, as indicated by the brown arrows sketched in Fig. 3b. Thus, the resulting flow speed rapidly decays along the $r$-direction, as compared to a pure system (Fig. $3 \mathrm{e}, \mathrm{f}$ and Supplementary Video 10).

We observe that the maximum Marangoni convective flow speed occurs in the replenishment zone $(r \approx R$ ) (Fig. 3e and Supplementary Videos 3 and 4 ). The location of the replenishment 
Table 1 | Experimental conditions for the quasi-steady velocity profile measurements by varying the dynamic viscosity $\left(\mu_{\mathrm{b}}\right)$ of the bath liquid and the surface tension difference $\left(\Delta \gamma=\gamma_{13}-\gamma_{12}\right)$ between two fully miscible liquids.

\begin{tabular}{|c|c|c|c|c|}
\hline Case & Drop liquid & Bath liquid & $\mu_{\mathrm{b}}(\mathrm{mPa} \mathrm{s})$ & $\Delta \gamma\left(\mathrm{mN} \mathrm{m}^{-1}\right)$ \\
\hline 1 & & DI water & 1.0 & 51.6 \\
\hline 2 & & DI water (75 wt\%) + glycerol (25 wt\%) & 2.0 & 49.7 \\
\hline 3 & & DI water (50 wt\%) + glycerol (50 wt\%) & 5.0 & 46.8 \\
\hline 4 & & DI water + SDS $(0.10 \mathrm{cmc})$ & & 47.9 \\
\hline 5 & Isopropanol (IPA) & DI water + SDS $(0.53 \mathrm{cmc})$ & & 26.3 \\
\hline 6 & & DI water + SDS $(0.95 \mathrm{cmc})$ & 1.0 & 20.2 \\
\hline 7 & & DI water + CTAB $(0.27 \mathrm{cmc})$ & & 36.2 \\
\hline 8 & & DI water + CTAB $(0.48 \mathrm{cmc})$ & & 25.6 \\
\hline 9 & & Dl water + CTAB $(0.68 \mathrm{cmc})$ & & 22.6 \\
\hline 10 & & & & 2.5 \\
\hline 11 & Tert-butanol & Methanol & 0.53 & 3.6 \\
\hline 12 & Ethanol & & & 1.5 \\
\hline
\end{tabular}

The experimental results are provided in Fig. 3.

zone $(R)$ and the maximum flow speed $(U)$ are influenced by multiple variables of the system including, for example, viscosities $\left(\mu_{[\mathrm{b}, \mathrm{d}]}\right)$, densities $\left(\rho_{[\mathrm{b}, \mathrm{d}]}\right)$, surface tensions $\left(\gamma_{[12,13]}\right)$, droplet volume $(V)$, and the diffusion coefficient $(D)$ between two miscible liquids, where the subscripts ' $b$ ' and ' $d$ ' indicate the bath and drop liquid, respectively.

To understand the transport mechanism, we identified the dominant physical mechanisms and performed a scaling analysis to predict finite $R$ and $U$ as well as the time to establish the quasisteady state. In this problem, viscous effects are negligible for the initial spreading of the deposited drop because the Reynolds number is much larger than unity, $\operatorname{Re}=\rho U R / \mu=O\left(10^{3}\right)$. Also, we observed that a finite droplet radius $(R)$ is quickly developed in 40-60 ms (Fig. 1c; see more details in Supplementary Fig. 6). Compared to the whole experimental time period (1-2s), the time to develop a static lens shape is much shorter than the other important timescales. Gravity is not important because of the small Bond number, Bo $=\Delta \rho g R h / \Delta \gamma=O\left(10^{-3}\right)$, where $\Delta \rho=\rho_{\mathrm{b}}-\rho_{\mathrm{d}}$ and $h$ is the thickness of the liquid lens (Fig. 2a), which is a function of time and space during the initial spreading (see Supplementary Fig. 7 and Supplementary Video 11). Eventually, the lens becomes nearly flat in $O(10 \mathrm{~ms})$. Thus, for the spreading mechanism, we can estimate the order of magnitude of the dominant forces. At the early times, the driving force/volume for radial spreading is mainly due to the surface tension difference $(\Delta \gamma / \ell h)$ and the retarding force/volume is dominated by inertial effects $\left(\rho_{\mathrm{d}} \ell / t^{2}\right)$, where $\ell$ is the time-dependent spreading radius (see Fig. $1 \mathrm{c}$ and Supplementary Fig. 6$)^{22}$. Therefore, a balance between these two effects gives

$$
\ell(t) \approx\left(\frac{\Delta \gamma}{\rho_{\mathrm{d}} h(t)}\right)^{1 / 2} t
$$

As discussed above, when $S \approx 0$ due to mixing, the drop stops spreading and takes the shape of a static lens, that is, $\ell(t) \rightarrow R$. How long does it take to establish this condition? As long as the drop spreads, a diffusion boundary layer of thickness $\delta_{\mathrm{D}}$ will develop between the two liquids. When $\delta_{\mathrm{D}} \approx h$ at the leading edge, we assumed that the spreading stops although the drop thickness decreases (Supplementary Fig. 6) due to liquid leakage caused by the Marangoni flow at the edge of the static lens $(r \approx R)$. We take $h \approx V /\left(\pi R^{2}\right)$ for a lens shape of the drop and $\delta_{\mathrm{D}} \approx(D t)^{1 / 2}$, where $D$ is the diffusion coefficient between the two liquids. To establish the quasi-static lens shape, the required time $\left(\tau_{\mathrm{D}}\right)$ is approximately $h^{2} / D$, where the Stokes-Einstein model was used for the diffusion coefficient (see Methods). Then, the required time is rescaled as $\tau_{\mathrm{D}} \approx V^{2} /\left(\pi^{2} R^{4} D\right)$. Based on this idea, we use equation (2) to estimate the finite radius of the liquid lens on a fully miscible liquid bath:

$$
R \approx\left(\frac{\Delta \gamma V^{3}}{\pi^{3} \rho_{\mathrm{d}} D^{2}}\right)^{1 / 8}
$$

After establishing the quasi-static lens shape, a quasi-steady Marangoni flow along the interface is observed. At the rim of the lens $(r \approx R)$, the balance between Marangoni convection and diffusion results in a viscous boundary layer of thickness $\delta_{v} \approx\left(\nu \tau_{v}\right)^{1 / 2}$, where $v\left(=\mu_{\mathrm{b}} / \rho_{\mathrm{b}}\right)$ is the kinematic viscosity and the relevant timescale $\tau_{v} \approx R / U$, where we next estimate the flow speed $U$. The Marangoni stress that acts over the spreading distance is balanced with the viscous stresses, that is, $\Delta \gamma / R \sim \mu_{\mathrm{b}} U / \delta_{\nu}$. Using these results and equation (3), we obtain the maximum Marangoni convection flow speed

$$
U \approx\left[\frac{\pi \rho_{\mathrm{d}}^{1 / 3} D^{2 / 3}(\Delta \gamma)^{5}}{\mu_{\mathrm{b}}^{8 / 3} \rho_{\mathrm{b}}^{8 / 3} V}\right]^{1 / 8}
$$

and the required time to establish the quasi-steady state for the flow

$$
\tau_{\nu} \approx\left[\frac{\mu_{\mathrm{b}}^{1 / 3} \rho_{\mathrm{b}}^{1 / 3} V^{1 / 2}}{\pi^{1 / 2} \rho_{\mathrm{d}}^{1 / 6} D^{1 / 3}(\Delta \gamma)^{1 / 2}}\right]
$$

Experimental and theoretical results are compared in Fig. 4. To check the theoretical model, we performed extensive experiments by varying the droplet size, liquid system, viscosity, density, diffusion coefficient, and surface tension, as summarized in Table 2 . The theoretical predictions are in good agreement with experimental results.

We have focused on the flows and spreading mechanisms of a fully soluble droplet on a liquid bath, which displays finite diffusion time and length scales at the interface of the two liquids. The surface tension difference triggers the Marangoni flow, $U \approx O\left(0.1 \mathrm{~m} \mathrm{~s}^{-1}\right)$, and it mixes the two materials (Supplementary Videos 3-7), which also apply to dynamics for surfactant-mediated delivery of medications for chronic lung disease $\mathrm{e}^{23}$ and eye disease $\mathrm{e}^{24}$. Although surfactants are a good material to deliver chemicals effectively ${ }^{25}$, the surfactants typically remain near the liquid-air interface ${ }^{21}$. However, as described here, a miscible solute causing solutal Marangoni flows will mix with a bulk liquid and does not significantly change the surface property. Nevertheless, the solutal Marangoni flow can not only deliver materials but also clean liquid surfaces without surface contamination (Supplementary Videos 7 and 9). For example, the deposited drop of solute spreads over an area of $30 \mathrm{~cm}^{2}$ in $200 \mathrm{~ms}$. This fundamental study for Marangoni flow phenomena is thus 

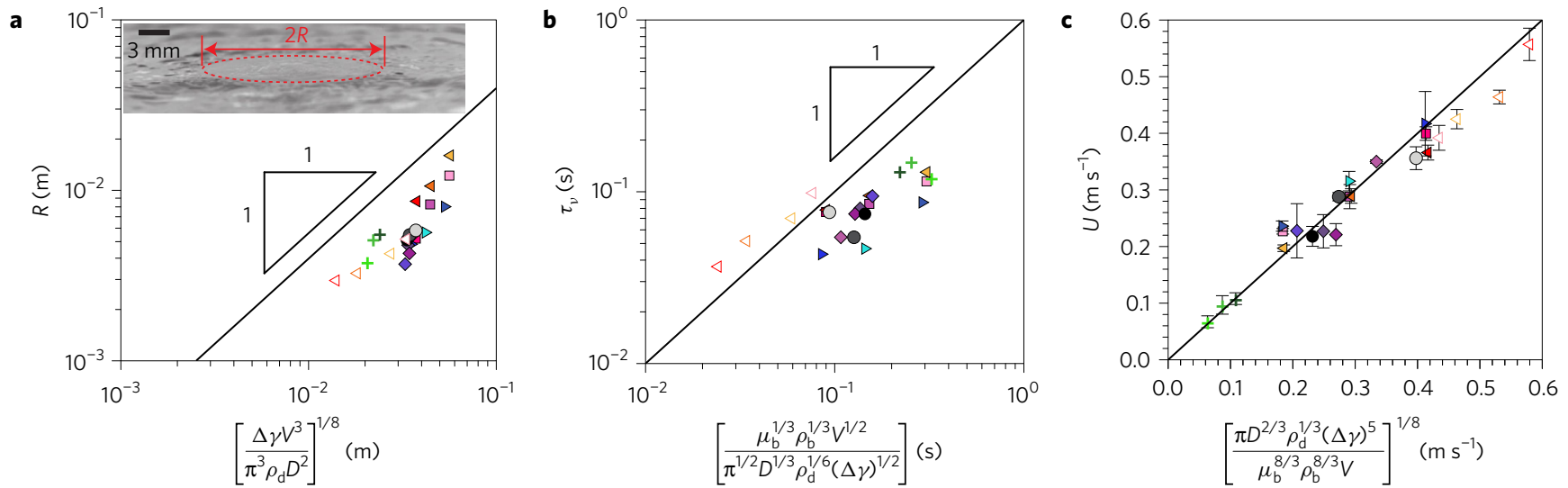

Figure 4 | Comparing theory and measurements for Marangoni-driven transport phenomenon involving fully miscible liquids. a, The finite spreading radius in the quasi-steady regime. Inset, the definition of the constant spread diameter $(2 R)$. $\mathbf{b}$, The timescale $\left(\tau_{v}\right)$ to reach the quasi-steady regime for the Marangoni flow. c, Maximum Marangoni convective flow speed (Fig. 3e). The error bars are determined from the standard deviation of the maximum velocity magnitudes during the quasi-steady regime. The symbols are summarized in Table 2.

Table 2 | Experimental conditions for comparing theory and measurement results for Marangoni-driven transport phenomena in fully miscible liquids.

\begin{tabular}{|c|c|c|c|}
\hline Symbol & Drop liquid & Drop volume $(\mu \mathrm{l})$ & Bath liquid \\
\hline & & 0.5 & \\
\hline & & 1.0 & DI water $(400 \mathrm{ml})$ \\
\hline & & 3.0 & \\
\hline & & 5.0 & \\
\hline & & & DI water $(400 \mathrm{ml})$ \\
\hline & & & DI water $(300 \mathrm{ml})+$ glycerol $(100 \mathrm{ml})$ \\
\hline & & & DI water $(200 \mathrm{ml})+$ glycerol $(200 \mathrm{ml})$ \\
\hline & Isopropanol (IPA) & & DI water $(400 \mathrm{ml})+$ CTAB (140 mg) \\
\hline & & & DI water $(400 \mathrm{ml})+$ CTAB $(100 \mathrm{mg})$ \\
\hline & & & Dl water $(400 \mathrm{ml})+\mathrm{CTAB}(70 \mathrm{mg})$ \\
\hline & & & DI water $(400 \mathrm{ml})+$ CTAB $(40 \mathrm{mg})$ \\
\hline & & & DI water $(400 \mathrm{ml})+\mathrm{SDS}(900 \mathrm{mg})$ \\
\hline & & & DI water $(400 \mathrm{ml})+\mathrm{SDS}(500 \mathrm{mg})$ \\
\hline & & 7.2 & DI water $(400 \mathrm{ml})+$ SDS $(100 \mathrm{mg})$ \\
\hline & & & Methanol (400 ml) \\
\hline & & & DI water $(400 \mathrm{ml})$ \\
\hline & Methanol & & DI water $(300 \mathrm{ml})+$ glycerol $(100 \mathrm{ml})$ \\
\hline & & & DI water $(200 \mathrm{ml})+$ glycerol $(200 \mathrm{ml})$ \\
\hline & & & Methanol (400 ml) \\
\hline & & & DI water $(400 \mathrm{ml})$ \\
\hline & Ethanol & & DI water $(300 \mathrm{ml})+$ glycerol $(100 \mathrm{ml})$ \\
\hline & & & DI water $(200 \mathrm{ml})+$ glycerol $(200 \mathrm{ml})$ \\
\hline & Tert-butanol & & Methanol (400 ml) \\
\hline
\end{tabular}

The results are provided in Fig. 4 
expected to affect material dissolution, transport and cleaning in a myriad of applications.

\section{Methods}

Methods, including statements of data availability and any associated accession codes and references, are available in the online version of this paper.

Received 23 December 2016; accepted 17 June 2017; published online 31 July 2017

\section{References}

1. Hoult, D. P. Oil spreading on the sea. Annu. Rev. Fluid Mech. 4, 341-368 (1972)

2. Blumer, M. \& Sass, J. Oil pollution: persistence and degradation of spilled fuel oil. Science 176, 1120-1122 (1972).

3. Fischer, H. B. Mixing and dispersion in estuaries. Annu. Rev. Fluid Mech. 8, 107-133 (1976)

4. Pyle, D. L., Fryer, P. J. \& Reilly, C. D. Chemical Engineering for the Food Industry (Springer Science \& Business Media, 2012).

5. Hashimoto, N., Ishii, H., Tanahashi, K., Ohno, K. \& Ohno, K. Fluid mixing element. US patent 4,884,894 (1989).

6. Rudick, A. G. Tri-mix sugar based dispensing system. US patent 4,753,370 (1988)

7. Stone, H. A., Stroock, A. D. \& Ajdari, A. Engineering flows in small devices: microfluidics toward a lab-on-a-chip. Annu. Rev. Fluid Mech. 36, 381-411 (2004).

8. Plochocki, A. P., Dagli, S. S. \& Andrews, R. D. The interface in binary mixtures of polymers containing a corresponding block copolymer: effects of industrial mixing processes and of coalescence. Polym. Eng. Sci. 30, 741-752 (1990).

9. Franklin, B., Brownrigg, W. \& Farish, Mr. Of the stilling of waves by means of oil. Phil. Trans. 64, 445-460 (1774).

10. Thoroddsen, S. T. \& Takehara, K. The coalescence cascade of a drop. Phys. Fluids 12, 1265-1267 (2000).

11. Sternling, C. V. \& Scriven, L. E. Interfacial turbulence: hydrodynamic instability and the Marangoni effect. AIChE J. 5, 514-523 (1959).

12. Schwarzenberger, K. et al. Pattern formation and mass transfer under stationary solutal Marangoni instability. Adv. Colloid Interface Sci. 206, 344-371 (2014)

13. Carter, E. G. \& Jones, D. C. An experimental test of the validity of Antonow's rule. Trans. Faraday Soc. 30, 1027-1038 (1934).

14. Roché, M. et al. Marangoni flow of soluble amphiphiles. Phys. Rev. Lett. 112, 208302 (2014).

15. Suciu, D. G., Smigelschi, O. \& Ruckenstein, E. The spreading of liquids on liquids. J. Colloid Interface Sci. 33, 520-528 (1970).
16. Ruckenstein, E., Smigelschi, O. \& Suciu, D. G. A steady dissolving drop method for studying the pure Marangoni effect. Chem. Eng. Sci. 25, 1249-1254 (1970).

17. Sada, E., Ameno, T. \& Ando, T. A study of interfacial flow due to concentration difference. Chem. Eng. Sci. 32, 1269-1275 (1977).

18. Dussaud, A. D. \& Troian, S. M. Dynamics of spontaneous spreading with evaporation on a deep fluid layer. Phys. Fluids 10, 23-38 (1998)

19. Walters, D. A. A stable transitional flow pattern in the surface tension driven spreading of ethanol-water solutions. Langmuir 6, 991-994 (1990).

20. Kim, H., Lee, J., Kim, T.-H. \& Kim, H.-Y. Spontaneous Marangoni mixing of miscible liquids at a liquid-liquid-air contact line. Langmuir 31, 8726-8731 (2015)

21. Phan, C. M. Dissociation of ionic surfactants at the air/water interface: complete or partial? J. Phys. Chem. B 120, 7681-7686 (2016).

22. Blanchette, F., Messio, L. \& Bush, J. W. M. The influence of surface tension gradients on drop coalescence. Phys. Fluids 21, 072107 (2009)

23. Grotberg, J. B. Pulmonary flow and transport phenomena. Annu. Rev. Fluid Mech. 26, 529-571 (1994)

24. Braun, R. J. Dynamics of the tear film. Annu. Rev. Fluid Mech. 44, 267-297 (2012)

25. Grotberg, J. B. Respiratory fluid mechanics and transport processes. Annu. Rev. Biomed. Eng. 3, 421-457 (2001).

\section{Acknowledgements}

H.K. thanks H.-Y. Kim for helpful discussions. O.S. thanks the Natural Sciences and Engineering Research Council of Canada (NSERC) for a postdoctoral fellowship. K.M. thanks the Justus and Louise van Effen Research Grant from TU Delft and Dr. Hendrik Muller Foundation for a Visiting Student Research Collaborator (VSRC) programme in Princeton University. We thank J. Nunes, A. Perazzo and S. Suin for helpful discussions.

\section{Author contributions}

H.K. conceived the project and planned the experiments. H.K. and K.M. built the experimental apparatus and carried out the experiments. H.K., O.S., S.A. and H.A.S. analysed the data and developed the theoretical analysis. H.K. wrote the first draft of the manuscript. All authors discussed and edited the manuscript.

\section{Additional information}

Supplementary information is available in the online version of the paper. Reprints and permissions information is available online at www.nature.com/reprints. Publisher's note: Springer Nature remains neutral with regard to jurisdictional claims in published maps and institutional affiliations. Correspondence and requests for materials should be addressed to H.K. or H.A.S.

\section{Competing financial interests}

The authors declare no competing financial interests. 


\section{Methods}

$400 \mathrm{ml}$ DI water is filled in a Petri dish (196 mm diameter, Himedia Lab.). The depth $(H)$ of the liquid bath is about $14 \mathrm{~mm}$ and the volume of the deposited drop is varied from $0.5 \mu \mathrm{l}$ to $7.2 \mu \mathrm{l}$. The $7.2 \mu \mathrm{l}$ droplet is generated at the tip of a needle (inner diameter $\approx 0.3 \mathrm{~mm}$ ) and the liquid is supplied by a syringe pump (Harvard PHD 2000). The needle tip is fixed $4 \mathrm{~mm}$ above the water surface. For the smaller droplets $(<7 \mu \mathrm{l})$, we carefully deposited liquid using a micropipette $(0.5-10 \mu \mathrm{l}$, Eppendorf Research). To minimize the inertial and evaporation effects, we used a micropipette to control the droplet volume.

DI water (resistivity $=18.2 \mathrm{M} \Omega \mathrm{cm}$, Milli-Q Millipore) has density $\rho=0.999 \mathrm{~g} \mathrm{~cm}^{-3}$, viscosity $\mu=1 \mathrm{mPa}$, and surface tension $\gamma=72.0 \mathrm{mN} \mathrm{m}^{-1}$. The deposited droplets are isopropanol (purity $=99.5 \%, \mathrm{BDH}, \mathrm{USA}$ ) $\left(\rho=0.785 \mathrm{~g} \mathrm{~cm}^{-3}, \mu=1.1 \mathrm{mPa}\right.$, and $\left.\gamma=21.2 \mathrm{mN} \mathrm{m}^{-1}\right)$, ethanol (anhydrous, 99.5\%, Sigma-Aldrich) ( $\rho=0.789 \mathrm{~g} \mathrm{~cm}^{-3}, \mu=1.07 \mathrm{mPa}$, and $\left.\gamma=22.1 \mathrm{mN} \mathrm{m}^{-1}\right)$, methanol (99\%, Sigma-Aldrich) $\left(\rho=0.792 \mathrm{~g} \mathrm{~cm}^{-3}, \mu=0.53 \mathrm{mPa}\right.$, and $\gamma=23.7 \mathrm{~m} \mathrm{~N} \mathrm{~m}^{-1}$ ), tert-butanol (anhydrous, $99.5 \%$, Sigma-Aldrich) $\left(\rho=0.775 \mathrm{~g} \mathrm{~cm}^{-3}, \mu=3.35 \mathrm{mPa}\right.$, and $\left.\gamma=20.1 \mathrm{mN} \mathrm{m}^{-1}\right)$, and $5 \mathrm{cSt}$ silicone oil (Sigma-Aldrich) $\left(\rho=0.913 \mathrm{~g} \mathrm{~cm}^{-3}, \mu=4.5 \mathrm{mPa} \mathrm{s}\right.$, and $\left.\gamma=21.2 \mathrm{mN} \mathrm{m}^{-1}\right)$.

For the case of fully miscible liquids, the diffusion coefficient is estimated by using the Stokes-Einstein model: $D=k_{\mathrm{B}} T /\left(6 \pi \mu_{\mathrm{b}} b\right)$, where $k_{\mathrm{B}}$ is Boltzmann's constant $\left(1.38 \times 10^{-23} \mathrm{~J} \mathrm{~K}^{-1}\right), T$ is the absolute temperature $(298 \mathrm{~K}), \mu_{\mathrm{b}}$ is the dynamic viscosity of the liquid bath, and $b$ is the radius of the molecule of the deposited liquid.

To study the effect of the liquid bath viscosity, glycerol $(99.7 \%, \mathrm{BDH})$ was mixed with DI water. The concentrations of glycerol in the water solution were 25 and $50 \mathrm{wt} \%$. The $25 \mathrm{wt} \%$ glycerol in the water mixture has $\rho=1.08 \mathrm{~g} \mathrm{~cm}^{-3}$, $\mu=2.02 \mathrm{mPa}$, and $\gamma=70.9 \mathrm{mN} \mathrm{m}^{-1}$. The $50 \mathrm{wt} \%$ glycerol in the water mixture has $\rho=1.13 \mathrm{~g} \mathrm{~cm}^{-3}, \mu=5.35 \mathrm{mPa}$, and $\gamma=68.0 \mathrm{mN} \mathrm{m}^{-1}$.

The surfactant used was cationic (cetyl trimethyl ammonium bromide; CTAB, 99\%, Amresco) or anionic (sodium dodecyl sulfate; SDS, 98.5\%, Sigma-Aldrich). The concentrations of surfactant solutions used for the bath in units of the $\mathrm{cmc}$ were $0.10\left(\gamma=69.1 \mathrm{mN} \mathrm{m}^{-1}\right), 0.53\left(\gamma=47.5 \mathrm{mN} \mathrm{m}^{-1}\right)$, and $0.95 \mathrm{cmc}$ $\left(\gamma=41.4 \mathrm{mN} \mathrm{m}^{-1}\right)$ for SDS and $0.27\left(\gamma=57.4 \mathrm{mN} \mathrm{m}^{-1}\right), 0.48\left(\gamma=46.8 \mathrm{mN} \mathrm{m}^{-1}\right)$, $0.68\left(\gamma=43.8 \mathrm{mN} \mathrm{m}^{-1}\right)$, and $0.96 \mathrm{cmc}\left(\gamma=37.9 \mathrm{mN} \mathrm{m}^{-1}\right)$ for CTAB, where the critical micelle concentrations of SDS and CTAB are $8.2 \mathrm{mM}$ and $1 \mathrm{mM}$, respectively.

To visualize the flow pattern, we used hydrophobic polystyrene tracer particles (density $\rho_{\mathrm{p}}=1.05 \mathrm{~g} \mathrm{~cm}^{-3}$ and diameter $d_{\mathrm{p}}=100 \mu \mathrm{m}$, Thermo Scientific) for the top-view measurements. In this study, the particle concentration is typically very low, that is, $N_{\mathrm{I}} \ll 1$ and $N_{\mathrm{s}} \ll 1$, where $N_{\mathrm{I}}$ and $N_{\mathrm{s}}$ are the typical image density and particle source density, respectively (see more details in the Supplementary Information $)^{26}$. For the side-view experiments, we seeded the particles in DI water after plasma treatment to modify the surface condition of the particle from hydrophobic to hydrophilic. For particle tracking velocimetry measurements, we assumed the particles closely follow the flow because the Stokes number $\left(S t=\tau_{\mathrm{p}} / \tau_{\mathrm{f}}\right)$ is much smaller than unity, where $\tau_{\mathrm{p}}=\left(\rho_{\mathrm{p}} d_{\mathrm{p}}^{2}\right) /(18 \mu)=O\left(10^{-4}\right)$ is the particle response time and $\tau_{\mathrm{f}}=(R / U)=O\left(10^{-1}\right)$ is the timescale of fluid motion. Here, $R$ is the radius of the static liquid lens and $U$ is the maximum Marangoni convective flow speed, which are obtained from the particle tracking velocimetry method.

For the top-view experiments, we used a high-speed CMOS camera (Phantom V7.3) having a pixel resolution of $800 \times 600$ and a 8-bit dynamic range at a frame rate of 4,000 fps. For side views, a high-speed CMOS camera (Phantom v9.1) is used, which has a pixel resolution of 1,634 $\times 400$ and a 8 -bit dynamic range at a frame rate of $400 \mathrm{fps}$. To capture the particle images in a specific plane, a thin light sheet of $1 \mathrm{~mm}$ is illuminated in the middle of the drop and the light sheet is generated by using a laser (wavelength $=520 \mathrm{~nm}$, power $=50 \mathrm{~mW}$, Coherent BioRay Laser) and cylindrical optics. For the details of the experimental set-ups, see Supplementary Information.

The physical properties were measured at $T=298 \mathrm{~K}$. We used a pendant droplet method to measure the surface tension values, which were computed by using an in-house Matlab code that is based on the algorithm of Rotenberg and colleagues $^{27}$. This code was validated by comparing with experimental results from a conventional goniometer (Theta Lite, Biolin Scientific). To measure the viscosities of all liquids, we used a rheometer (Anton-Paar MCR 301) with a CP50-1 geometry and a sandblasted cylinder system (CC27 geometry). Weights were measured by a Mettler Toledo XS105 scale.

Data availability. The data that support the plots within this paper and other findings of this study are available from the corresponding authors upon reasonable request.

\section{References}

26. Adrian, R. J. \& Westerweel, J. Particle Image Velocimetry (Cambridge Univ. Press, 2011).

27. Rotenberg, Y., Boruvka, L. \& Neumann, A. W. Determination of surface tension and contact angle from the shapes of axisymmetric fluid interfaces. J. Colloid Interface Sci. 93, 169-183 (1983). 